\title{
Design of a Low Cost Four-Channel LDR Based Line-Follower Sensor with Transient and External Interference Compensations
}

\author{
Guilherme M. Maciel* Iago Z. Biundini* Milena F. Pinto** \\ Ivo C. S. Junior* Andre L. M. Marcato* Adolfo G. Filho*** \\ * Faculdade de Engenharia Elétrica, Universidade Federal de Juiz de \\ Fora, Brasil. \\ ** Centro Federal de Educação Tecnológica Celso Suckow da Fonseca, \\ Brasil \\ *** Colégio Militar de Juiz de Fora, Brasil
}

\begin{abstract}
A line follower robot is a mobile robot that detects and follows a line drawn on the floor. A vast number of works proposed different sensing solutions for recognizing and following a line path, such as phototransistors, cameras, and infrared sensors. However, only solutions with low computational burden are adequate in case of limited processing, and low-cost requirement. The main contributions of this research are the implementation of a four-channel LDR based on a line-follower sensor and a novel methodology for recognizing colors at high speed with these LDRs. This proposed low-cost equipment enables the robot to identify lines of different colors and external illumination. The main advantage of the proposed solution is the simplicity and low computational burden that allows its use in a low-cost microcontroller and for educational purposes. The results have shown the applicability of the proposed solution for identifying and tracking a line path.
\end{abstract}

Keywords: Educational Robotics, Low-cost Sensor, Line Follower Robot, Instrumentation, Light Dependent Resistor.

\section{INTRODUCTION}

There are several different types of navigation systems in mobile robot paradigm. A line follower robot is a robot designed to follow a line or path already selected by the user and can be applied in a wide range of areas, such as industrial, domestic or health environments (Singh and Chouhan, 2017). A different and interesting application of a line follower robot is presented in Rafi et al. (2016), where the robotic system is applied to solve irrigation problems in developing countries. As defined in Sonal et al. (2017), this kind of system can follow a line using an onboard control circuit accurately, making them extremely useful when the path is well established.

The primary basic operations of a line follower robot are summarized as follows. First, the robot captures the line position through sensors mounted at its frame. Note that this requires high resolution and high robustness. Then, the robot should track the line with a steering mechanism and compensation to stabilize tracking motion. Finally, the robot must control the speed according to the lane condition.

The line sensing process requires high resolution and high robustness (Ghani et al., 2011). The solution to this problem is the use of a solid white line on a black floor (or black line on the white floor), which limits the information collected by the sensors (Hasan et al., 2012). However, in some applications, there is a need to identify colors for achieving different goals. There is a distinct kind of sensors that the robot can use for recognizing and following a line path. Among them can be cited the infrared ray sensors, cameras (Gomes et al., 2016), and phototransistors (Khade et al., 2017).

The work of Putra et al. (2017) developed a prototype of a line following robot using only visual information. The scenario is represented by a self-driving car as motivation. Image processing has a high computational burden, although the camera information is a useful feature source. It requires more powerful hardware to use computer vision libraries, such as OpenCV (Bradski, 2000). The camera is a solution with a high cost that depends on more sophisticated algorithms and difficult to apply in some environments.

The work of Singh and Chouhan (2017) applied infrared reflective sensors to estimate the deviation circular arc from a straight line. Based on that, the system determines the radius of the path and, then, the angular velocities of the two wheels of a differential robot. In the work of Sonal et al. (2017), the authors discuss the design of a sensor array using RGB LED as a transmitter, and a phototransistor as a receiver. The color recognition is performed through LDR sensors. However, it only detected the primary colors. A similar proposition is presented by Nugraha et al. (2015). The authors applied LDR color sensors, and the classification is performed by a fuzzy logic method using the voltage output from the LDR. 
A famous application of a line follower robot is in the RoboCup Junior Rescue (Sklar, 2004). In this competition, the robots must identify victims within re-created disaster scenarios, where the mission complexity is varied from line-following on a flat surface to paths through obstacles on uneven terrain. Figure 1 shows an example of a configuration for the rescue experiment using this kind of navigation system. Note that the colors on the ground represent the path. They are used for the robot's decisionmaking system. The robot must identify the line to follow from black and white colors. The green markers point the direction to follow. The gray one represents the entrance of the rescue area. The black marker indicates the victim localization.

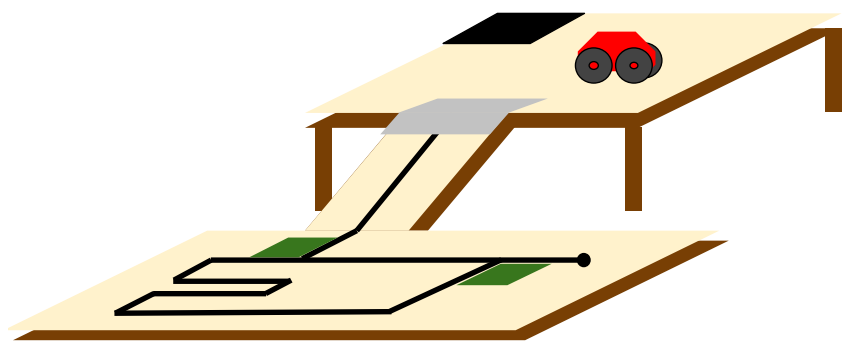

Figure 1. Example Configuration For the Rescue Experiment Using a Line Follower Robot System.

Another important application is in the educational robotics process. This application is a growing trend (Merino et al., 2016) due to the benefits of robotics in education, being an interdisciplinary subject with math, electronics, physics, among others (Greenberg et al., 2019). A drawback in engineering education is the difficulty of absorbing the rapid technological evolution of systems and equipment, especially in the areas of significant technological development (da Silva and de Assis Rangel, 2011). As an example, it can be cited the communication ports of computers that were previously parallel or serial ports. Nowadays, many computers only have USB type ports, which means that commercial equipment used as a teaching module can become obsolete quickly and disposable.

According to Cordeiro et al. (2009), the educational tools have the advantage of not being a proprietary system, i.e., it allows the student to know the real operation of the electronic circuits that involve the project. Another characteristic associated with the construction of this type of equipment is the flexibility of updating and modifying the firmware and hardware, which is a requirement due to the technological evolution. Based on this idea, this article presents the development of low-cost equipment to be used in a line follower robot as well as an educational module for engineering education.

\subsection{Main Contributions}

The main contribution of this work is the design and implementation of a four-channel LDR based line-follower sensor to be applied in mobile robots mission. This lowcost equipment enables the robot to recognize lines of different colors to make the required decisions for the RobotCup Junior Rescue competition. Another significant contribution is in the aspect of open hardware, which may contribute to engineering education. Other contributions of this work are divided among application, design, and implementation as follows.

- A low-cost and simple equipment for the use of a line follower robot, with an educational emphasis;

- A methodology that allows the use of LDR for identification of colors in high frequency, even with variations of external illumination and microcontroller processing limitations.

\subsection{Organization}

The remainder of the paper is organized as follows. Section 2 presents the project development and its mathematical foundations. The details of the environment and the devices used in the project are shown in Section 3. Section 4 presents the proposed experiments with a proper discussion of the results. The concluding remarks and future work are conducted in Section 5.

\section{PROJECT DEVELOPMENT}

Figure 2 presents a global overview of the proposed methodology. Firstly an LDR is excited by a cyclic process of triggering colored LEDs through the reflection of light on the ground. The produced analog signal passes through inverse low-pass filtering that tends to compensate for the LDR response delay. The signal is segmented into six variables according to LED triggering. Finally, a perceptron algorithm maps the six variables in the components in the RGB components, normalizing and compensating the external illumination.

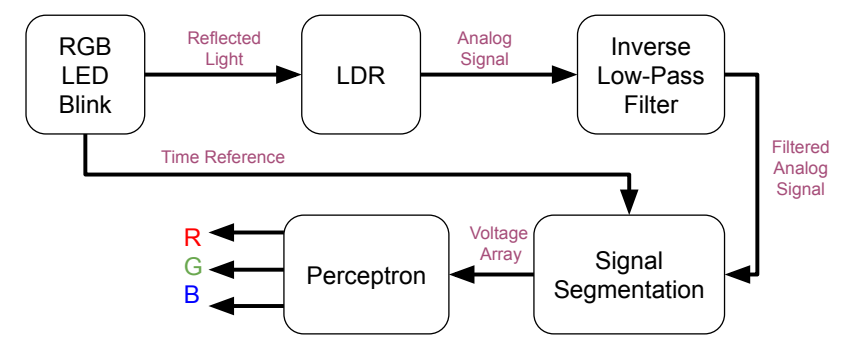

Figure 2. Overview of the Proposed Methodology.

\subsection{LDR Response Modeling}

LDR electrical resistance varies with respect to the luminous intensity that reaches it. The major problem of its use is the delayed response, which can be unfeasible for controller systems. The values can take almost a second to stabilize after a variation .

In this work, the LDR sensor circuit output was modeled as an approximation of a first-order low pass voltage filter to compensate for the negative effects of the delay. Equation 1 shows this compensation, where $K$ is the gain, and $\tau$ is the time constant.

$$
H(s)=K \frac{1}{\tau s+1}
$$


Applying the Z-transform with a sampling period $T$, and considering the gain of the filter with a unit value Rowell (2008), we have:

$$
H(z)=\frac{\left(1-e^{-\frac{T}{\tau}}\right) z^{-1}}{1-e^{-\frac{T}{\tau}} z^{-1}}
$$

Converting to a difference equation and isolating the input:

$$
x_{[n]}=\frac{y_{[n]}-e^{-\frac{T}{\tau}} y_{[n-1]}}{1-e^{-\frac{T}{\tau}}}
$$

Equation 3 performs the opposite of a first-order low pass filter. In this work, voltages are measured in a voltage divider circuit. It is possible to estimate the value that the sensor is reducing the latency effect with the correction of Equation 3. This process allows working with higher sampling frequencies, wich necessary for the control of the line follower.

\subsection{Color Sensing Process}

With the analog signals being filtered online by the Equation 3 , there is more sensitive information for rapid input variations. The next step is to process LDR1 and LDR4 signals and RGB LEDs for color discrimination on the ground.

Sampling Technique Similar to other related works, the principle of the color discrimination will be to measure the voltage response in the LDR circuit to different stimulus from the color switching of The RGB LED. The LED light strikes the surface, and the reflected portion of the LDR depends on the involved colors. This process is presented in Figure 3.
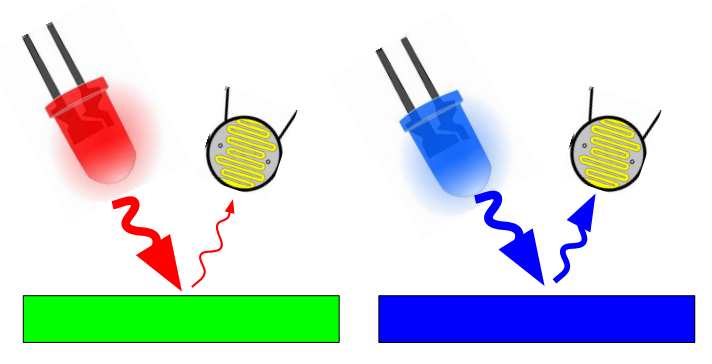

Figure 3. Sensor Operation.

The proposed technique consists of six voltage values $\boldsymbol{V}=\left[V_{0}, \ldots, V_{5}\right]$ which are continuously updated in a cyclical process. These voltages come from the AnalogDigital Converter signal filtered by Equation 3. Among them, the status of the LED changes between off and color: red, green, or blue. Figure 4 shows one cycle, which has a duration of six times the sampling period $t_{s}$. This values will generate a color output.

The decision to turn off the LED after each color activation is to increase contrast and help to compensate variations of ambient lighting. When the RGB LED is off, the LDR signal contains only residual voltage and external light interference. These are essential information for a robust color sensor.

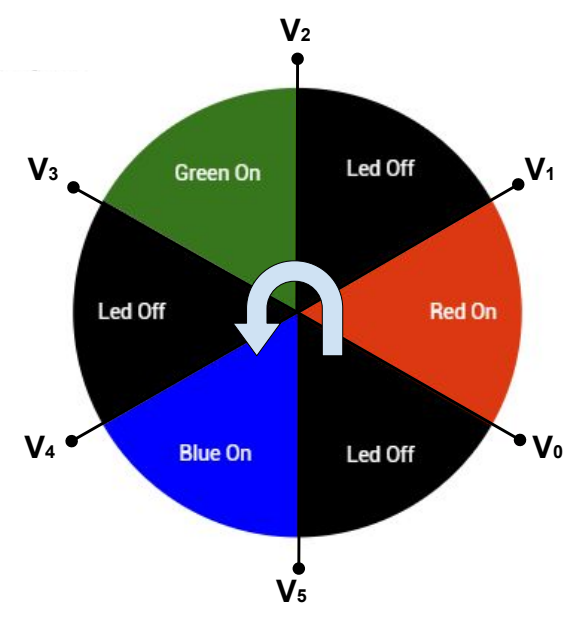

Figure 4. Sampling Cycle Process.

Color Components Extraction The voltage vector $\boldsymbol{V}$ is updated continuously. It is necessary a process to map the six values in the three color components. Thus, a linear perception was trained to make this mapping. The choice of the linear relationship is to get the maximum speed on limited hardware, such as an Arduino Mega. According to Figure 5, the inputs are the six voltages of $\boldsymbol{V}$, and the outputs are the normalized Red, Green, and Blue components.

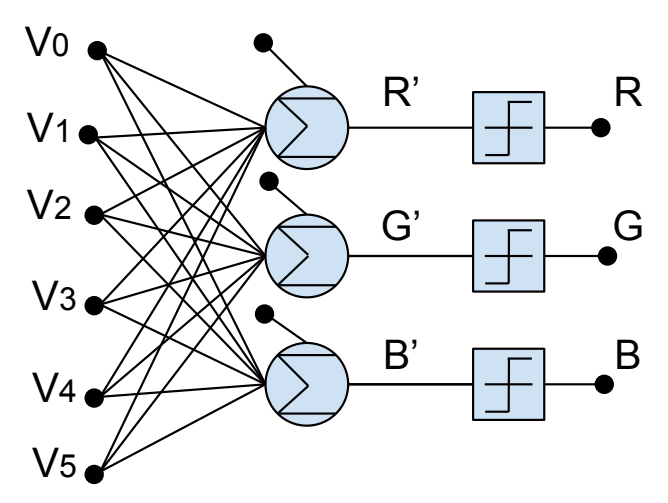

Figure 5. Schematic of the Linear Classifier Perceptron.

The perceptron process consists in a linear regression between $\boldsymbol{V}$ and $R^{\prime}, G^{\prime}$ and $R^{\prime}$ followed by a saturation between zero and one, getting $R, G$, and $B$. It is necessary to collect supervised data to obtain the linear correlation matrix $\boldsymbol{X}$ of each color sensor (see Equation 4). Data sets were collected from five different tonnes.

These data consist of the six voltage values of each situation. Note that is desired robustness in the variation of ambient light. Thus, the data were collected in three different environments: (i) No lighting; (ii) Office light; and (iii) indirect daylight. Table 1 shows the colors, the expected RGB of each one, and data training length.

$$
\left[\begin{array}{lllllll}
V_{0} & V_{1} & V_{2} & V_{3} & V_{4} & V_{5} & 1
\end{array}\right] \boldsymbol{X}=\left[\begin{array}{lll}
R^{\prime} & G^{\prime} & B^{\prime}
\end{array}\right]
$$

The pseudo-inverse least-square method was used to compute $\boldsymbol{X}$ (Equation 7 ). The $n$ collected data and the corre- 
Table 1. Training Colors

\begin{tabular}{ccccc}
\hline Color & R & G & B & Data Points \\
\hline Black & 0 & 0 & 0 & 300 \\
White & 1 & 1 & 1 & 300 \\
Red & 1 & 0 & 0 & 300 \\
Green & 0 & 1 & 0 & 300 \\
Blue & 0 & 0 & 1 & 300 \\
\hline
\end{tabular}

sponded expected RGB values are merged as presented in Equations 5, 6 and 7.

$$
\begin{gathered}
\boldsymbol{A}=\left[\begin{array}{ccccccc}
V_{0,1} & V_{2,1} & V_{3,1} & V_{4,1} & V_{5,1} & V_{6,1} & 1 \\
V_{0,2} & V_{1,2} & V_{2,2} & V_{3,2} & V_{4,2} & V_{5,2} & 1 \\
\vdots & & & \vdots & & & \vdots \\
V_{0, n} & V_{1, n} & V_{2, n} & V_{3, n} & V_{4, n} & V_{5, n} & 1
\end{array}\right] \\
\boldsymbol{B}=\left[\begin{array}{ccc}
R_{1} & G_{1} & B_{1} \\
R_{2} & G_{2} & B_{2} \\
\vdots & & \vdots \\
R_{n} & G_{n} & B_{n}
\end{array}\right] \\
\boldsymbol{X}=\left(A^{T} A\right)^{-1} A^{T} B
\end{gathered}
$$

\section{MATERIALS DESCRIPTION}

\subsection{Circuit Design}

The proposed circuit presents four LDR channels. They are responsible for identifying the color features on the ground of the competition. Due to the characteristics of the competition, the correct positioning of these sensors is crucial. In the developed circuit, the LDRs were positioned, as shown in Figure 6. They can be divided into two groups:

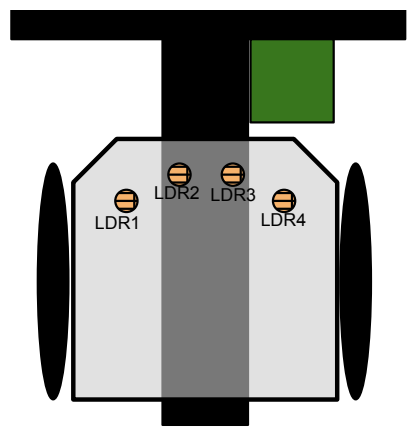

Figure 6. LDR Positioning

- Middle LDRs: The LDR2 and LDR3 are positioned in the front center of the circuit. They act as reflectance sensors, differentiating shades of gray. They are responsible for keeping the robot on the black line. If one of them stops being on the line, a smooth adjustment of the trajectory is made to correct it;

- Lateral LDRs: LDR1 and LDR4 are fixed on the laterals back. They act as color sensors, i.e., recognizing the RGB components of the surface. They are responsible for identifying the green markers as well as for helping the system on sharp curves, where the central LDRs go off the line.
Figure 7 shows the onboard hardware schematic. The LEDs provide a controlled stimulus of radiation for LDRs to measure their reflection on the ground. The central LDRs are accompanied by white LEDs, as well as the sides by RGB LEDs. There are three transistors to trigger each component of the RGB LEDs individually. A set of resistors help in the instrumentation and the activation of the LEDs and transistors. On the pins, $V C_{2}$ and $G N D$, it is applied 5 volts. $P_{2}, P_{3}$, and $P_{6}$ are connected to digital ports to drive the transistor. Finally, $P_{1}, P_{4}, P_{5}$, and $P_{7}$ are related to the analog inputs.

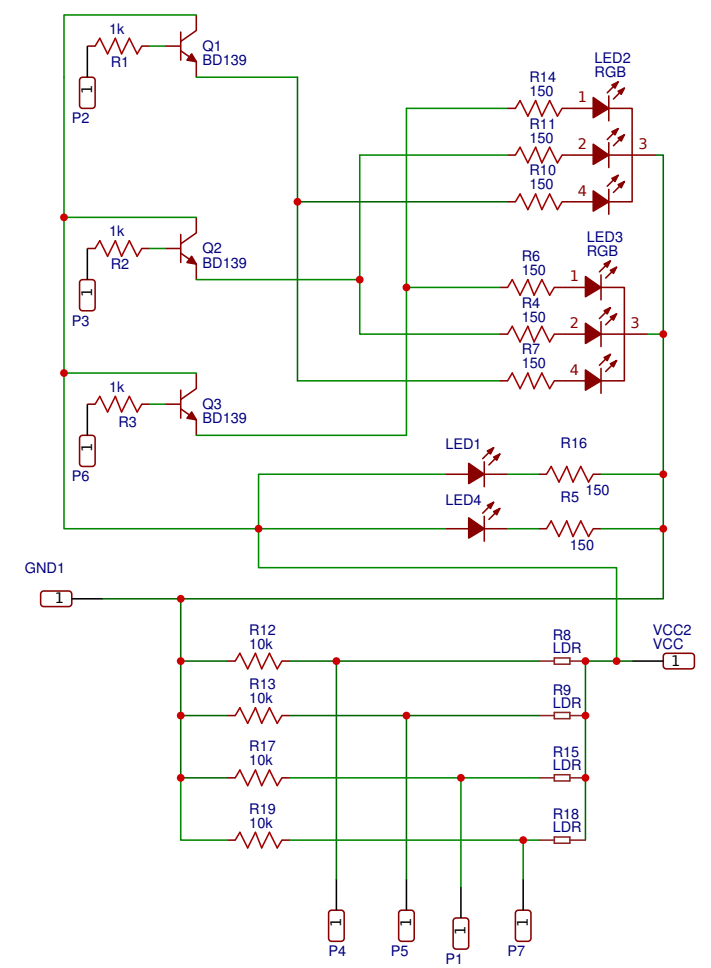

Figure 7. Circuit Diagram.

Table 2 exposes the components and their approximate commercial prices in Brazil in 2019. The circuit can be easily made in a Printed Circuit Board (PCB). Figure 8 shows the PCB with the components location.

Table 2. Components of the Project.

\begin{tabular}{ccc}
\hline Component & Amount & Price(R\$) \\
\hline Resistor 1/8 W & 15 & 1,00 \\
LDR 5mm & 4 & 1,20 \\
Transistor BC368 & 3 & 1,50 \\
White LED & 2 & 0,50 \\
RGB LED & 2 & 1,60 \\
PCB Board & 1 & 1,20 \\
\hline Total & 27 & 7,00 \\
\hline
\end{tabular}

\section{RESULTS AND DISCUSSIONS}

The tests were performed using a microcontroller Arduino Mega board. In a dedicated application, it was possible to exceed $1500 \mathrm{~Hz}$ sampling rate. But, as in Robocup Junior Robots competition requires other types of sensing, actuators, and controls, the rate was set at $500 \mathrm{~Hz}$, i.e., 


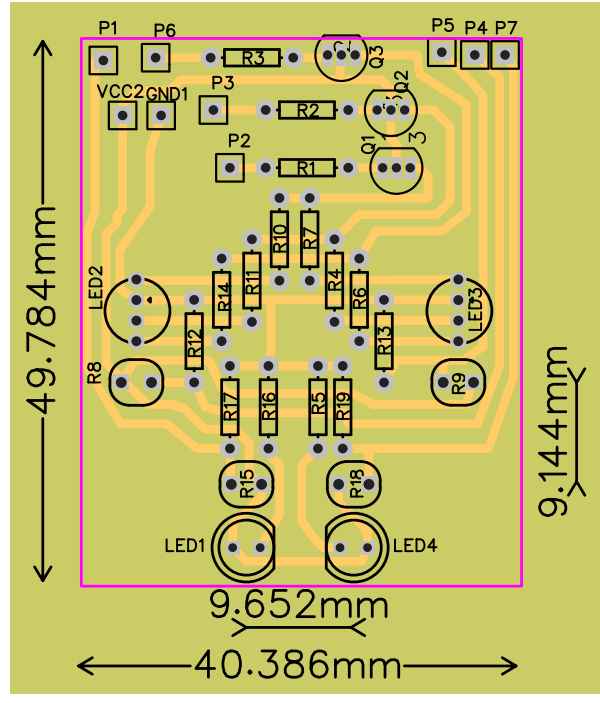

Figure 8. PCB Circuit.

$T=0.002 \mathrm{~s}$. At this frequency, a robot at $15 \mathrm{~cm} / \mathrm{s}$ could obtain approximately sixty measurements on a width of insulation tape, totalizing ten complete cycles of $V$. It is worth to mention that through the tests, the LDRs were approximated by a first-order filter with a time constant of $0.01 \mathrm{~s}$. A high value compared to the sampling time of 0.002 seconds.

Figure 9 shows the robotic platform that the sensor is fixed. It is a four-wheel differential drive with $3-6 \mathrm{~V}$ motors. The lower part of the sensor is a little more than $1 \mathrm{~cm}$ off the ground because there are bumpers in the competition.

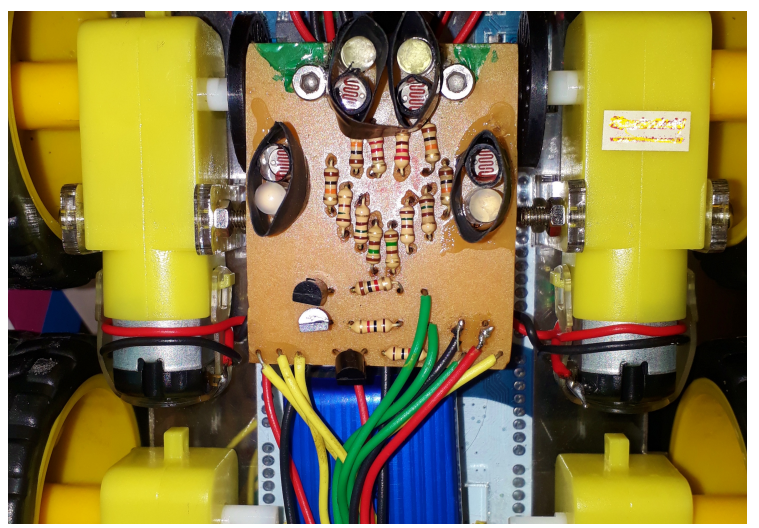

Figure 9. Circuit Under the Mobile Robot.

In the color specter test, the robot is programmed to move over a colored paper (Figure 10), saving in the log the recognized colors in the path.

The robot accelerated to a maximum of $1200 \mathrm{~ms}$ starting from the color sensors on the black stripe. The robot finishes the course on the white paper. Note an excellent reproduction of the bands and the colors in spite of a small mixture of tonality in the edges.

Another important test is concerning the abrupt variation of the environment light. Figure 12 shows the results. In the experiment, there was a sudden variance of external illumination through a high-flashlight. This interference

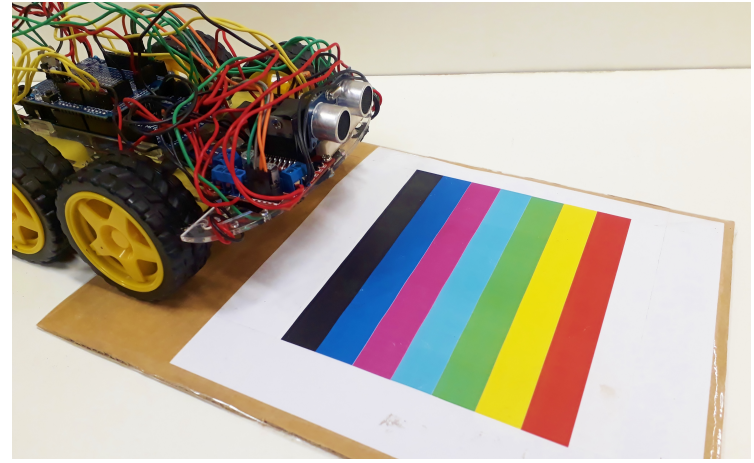

Figure 10. Setup of the test and the robot used in the experiment.
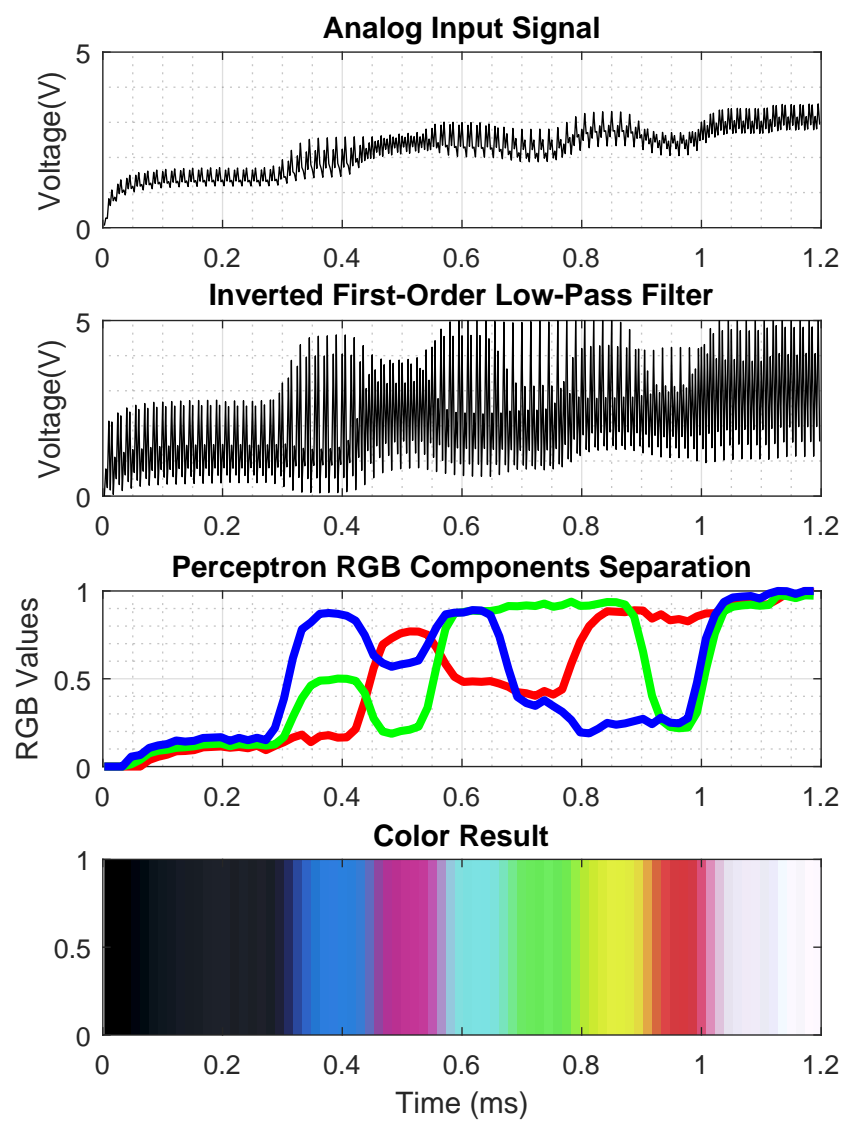

Figure 11. Experiment with Abrupt Colors Variation.

almost doubled the input voltage, but there was only a slight change in the output color bright.

The operational results of the proposed sensor can also be found in https://youtu.be/d_OC2dfmGic. In these experiments, the sensor is continuously recognizing the green markings to take the right path.

\section{CONCLUSION AND FUTURE WORK}

This research presented a piece of low-cost equipment that enables a line follower robot to recognize lines of different colors to make the required decisions. The proposed hardware is a four-channel, LDR based line-follower sensor. The 

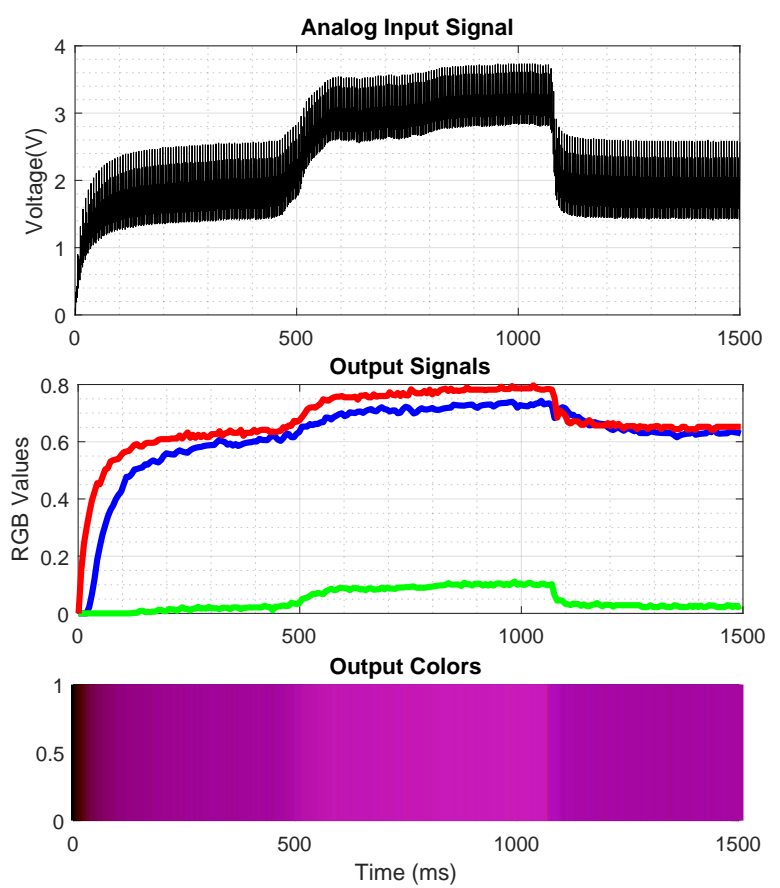

Figure 12. Experiment with Abrupt Variation of External Lighting.

main advantages of this kind of sensor is the simplicity and low computational burden that enables its applicability with a low-cost microcontroller, which can be used for educational purposes.

The experiments confirmed the successful use of the proposed solution for identifying and tracking a line path to be applied in line follower mobile robot. The processing velocity of both the sensor and microcontroller were adequate for the proposed task. This work opens the possibility of several future developments, such as the development of an educational tool to be used in robotics classes. For instance, the students could change some algorithm, such as the classification method, e.g., Fuzzy or Support Vector Machine (SVM). Besides, the experiment should be tested in more different scenarios to test its robustness.

\section{ACKNOWLEDGMENT}

Authors would like to thank the Universidade Federal de Juiz de Fora, Clube de Robótica - Colégio Militar de Juiz de Fora, CEFET-RJ, Rinobot Team, CAPES, FAPEMIG, INERGE, ANEEL and CNPq for all financial, technical and scientific support on this work.

\section{REFERENCES}

Bradski, G. (2000). The OpenCV Library. Dr. Dobb's Journal of Software Tools.

Cordeiro, A., Foito, D., and Guerreiro, M. (2009). Power electronics didactic modules for direct current machine control. In 2009 International Conference on Power Engineering, Energy and Electrical Drives, 635-639. IEEE.

da Silva, T.M.P. and de Assis Rangel, J.J. (2011). Discrete event simulation as didactic support to the teaching of telecommunications systems: applications in digital telephony. In Proceedings of the 2011 Winter Simulation Conference (WSC), 3888-3898. IEEE.

Ghani, N.M.A., Naim, F., and Yon, T.P. (2011). Two wheels balancing robot with line following capability. World Academy of Science, Engineering and Technology, 55, 634-638.

Gomes, M., Bássora, L., Morandin, O., and Vivaldini, K.C.T. (2016). Pid control applied on a line-follower agv using a rgb camera. In 2016 IEEE 19th International Conference on Intelligent Transportation Systems (ITSC), 194-198. IEEE.

Greenberg, R.I., Thiruvathukal, G.K., and Greenberg, S.T. (2019). Integrating mathematics and educational robotics: Simple motion planning. In Proceedings of the 10th International Conference on Robotics in Education (RiE 2019).

Hasan, K.M., Al Mamun, A., et al. (2012). Implementation of autonomous line follower robot. In 2012 International Conference on Informatics, Electronics \& Vision (ICIE V), 865-869. IEEE.

Khade, K., Naik, R., and Patil, A. (2017). Design of all color line follower sensor with auto calibration ability. In 2017 7th International Symposium on Embedded Computing and System Design (ISED), 1-5. IEEE.

Merino, P.P., Ruiz, E.S., Fernandez, G.C., and Gil, M.C. (2016). A wireless robotic educational platform approach. In 2016 13th International Conference on Remote Engineering and Virtual Instrumentation (REV), 145-152. IEEE.

Nugraha, M., Ardianto, P.R., and Darlis, D. (2015). Design and implementation of rfid line-follower robot system with color detection capability using fuzzy logic. In Control, Electronics, Renewable Energy and Communications (ICCEREC), 2015 International Conference on, 75-78. IEEE.

Putra, M.A., Pitowarno, E., and Risnumawan, A. (2017). Visual servoing line following robot: Camera-based line detecting and interpreting. In Engineering Technology and Applications (IES-ETA), 2017 International Electronics Symposium on, 123-128. IEEE.

Rafi, R.H., Das, S., Ahmed, N., Hossain, I., and Reza, S.T. (2016). Design \& implementation of a line following robot for irrigation based application. In Computer and Information Technology (ICCIT), 2016 19th International Conference on, 480-483. IEEE.

Rowell, D. (2008). 2.161 signal processing: Continuous and discrete, fall 2008.

Singh, J. and Chouhan, P.S. (2017). A new approach for line following robot using radius of path curvature and differential drive kinematics. In Computer Applications In Electrical Engineering-Recent Advances (CERA), 2017 6th International Conference on, 497502. IEEE.

Sklar, E. (2004). A long-term approach to improving human-robot interaction: Robocupjunior rescue. In Robotics and Automation, 2004. Proceedings. ICRA'04. 2004 IEEE International Conference on, volume 3, 2321-2326. IEEE.

Sonal, G., Raninga, P., and Patel, H. (2017). Design and implementation of rgb color line following robot. In Computing Methodologies and Communication (ICCMC), 2017 International Conference on, 442-446. IEEE. 